\title{
Exciton supersolidity in hybrid Bose-Fermi systems
}

\author{
Michał Matuszewski \\ Instytut Fizyki PAN, Aleja Lotników 32/46, 02-668 Warsaw, Poland \\ Thomas Taylor and Alexey V. Kavokin \\ School of Physics and Astronomy, University of Southampton, Southampton, SO171BJ, United Kingdom
}

\begin{abstract}
We investigate the ground states of a Bose-Einstein condensate of indirect excitons coupled to an electron gas. We show that in a properly designed system, the crossing of a roton minimum into the negative energy domain can result in the appearance of the supersolid phase, characterized by periodicity in both real and reciprocal space. Accounting for the spin-dependent exchange interaction of excitons we obtain ferromagnetic supersolid domains. The Fourier spectra of excitations of weakly perturbed supersolids show pronounced diffraction maxima which may be detected experimentally.

PACS numbers: 03.75.Hh, 05.30.Fk, 73.22.Lp, 78.67.Pt
\end{abstract}

A supersolid phase is characterised by superfludity and long range order in both real and reciprocal spaces [1]. One of the suggested indications of this phase transition is a "rotonlike instability" observed if the energy of the roton minimum of a superfluid is pushed below the energy of the ground state (characterised by zero wave-vector, q) [2]. Strong indications of the phase tranistion to supersolidity have been found in ${ }^{4} \mathrm{He}$ at low temperatures and high pressure 3]. Until now, experimental studies of supersolidity have been limited to ${ }^{4} \mathrm{He}$ and ${ }^{3} \mathrm{He}$, while, potentially, this phase may occur in any system of interacting bosons [4]. Here we discuss the possibility of formation of exciton supersolids in specially designed semiconductor structures.

Recently, Bose-Einstein condensation (BEC) and superfluidity of crystal quasiparticle excitations - excitons or exciton polaritons - have been reported in various semiconductor systems [5] 8 . The exciton-exciton interaction is usually almost independent of the wave vector [9], which is probably why in most experimentally studied cases the roton minimum is not formed. Nevertheless, formation of a roton minimum and even of a roton instability has been predicted for a hybrid system where a two-dimensional (2D) exciton condensate is placed in close vicinity to a $2 \mathrm{D}$ electron gas [10]. Moreover, the recent theoretical paper by Parish et al [11] evokes the possibility of the supersolid phase for excitons in asymetrically populated bilayers. Here we show that an exciton condensate in a hybrid system where excitons and an electron gas interact but do not overlap can undergo a transition to the supersolid phase.

We shall consider a system of coupled semiconductor quantum wells (CQW) which contain the BEC of spatially indirect excitons which has been widely studied in the past [12]. We introduce an important new element to this system: a 2D gas of free electrons located in a thin metallic layer grown on the top of semiconductor structure. The metal is separated from CQW by a wide band gap semiconductor layer, so that there is no overlap between free carriers and excitons, while they are close enough to each other to allow for efficient Coulomb interaction. The system is biased in such a way that the exciton dipole moments are oriented towards metal. This configuration prevents attraction between excitons and electrons and formation of trions. We phenomenologically describe spin-dependent interactions of dipole excitons by two constants, as specified below, and concentrate on the new physics brought by exciton-electron interactions. The system we study has been proposed for realization of charged electron-hole complexes [13] and of superconductivity mediated by the exciton condensate [14]. Compared to the unequally populated electronhole bilayers considered by Parish et al [1] this system has an evident advantage of protecting excitons from the phase space filling effect, which would allow operation with much higher exciton and 2D electron gas densities than in any system where excitons and free carriers spatially overlap. In the present work we concentrate on the phase trasitions of the exciton condensate induced by the presence of an electron gas in the neighboring metallic layer. Referring to the most popular GaAs based system, we account for the exchange interaction between excitons having +1 and -1 spin projections to the structure axis. This interaction, which represents the superexchange via intermediate dark excitons with spin projections +2 and -2 , has been recently addressed theoretically [15, 16].

An exciton condensate is described by a factorized Hartree-Fock ansatz $\Psi_{ \pm}\left(\mathbf{r}_{1}, \ldots, \mathbf{r}_{N}, t\right)=$ $\prod_{i} \psi_{ \pm}\left(\mathbf{r}_{i}, t\right)$ [17], which, in the limit of large number of particles $N$, leads to the following mean field energy with a nonlocal interaction potential, where \pm denotes the +1 and -1 exciton spin states,

$$
\begin{aligned}
& H_{ \pm}=N_{ \pm} \int d \mathbf{r} \psi_{ \pm}^{*}(\mathbf{r}, t)\left(-\frac{\hbar^{2}}{2 m_{\mathrm{ex}}} \nabla^{2}+\right. \\
& \left.+\frac{1}{2} \int d \mathbf{r}^{\prime}\left(V_{\mathrm{ex}-\mathrm{ex}}^{\mathrm{eff}}\left(\mathbf{r}-\mathbf{r}^{\prime}\right) n_{\mathrm{tot}}\left(\mathbf{r}^{\prime}, t\right)+\alpha n_{\mp}\left(\mathbf{r}^{\prime}, t\right)\right)\right) \psi_{ \pm}(\mathbf{r}, t)
\end{aligned}
$$

$\alpha$ denotes the interaction constant between the two exciton spin components, $n_{\text {tot }}(\mathbf{r}, t)=n_{+}(\mathbf{r}, t)+n_{-}(\mathbf{r}, t)=$ 

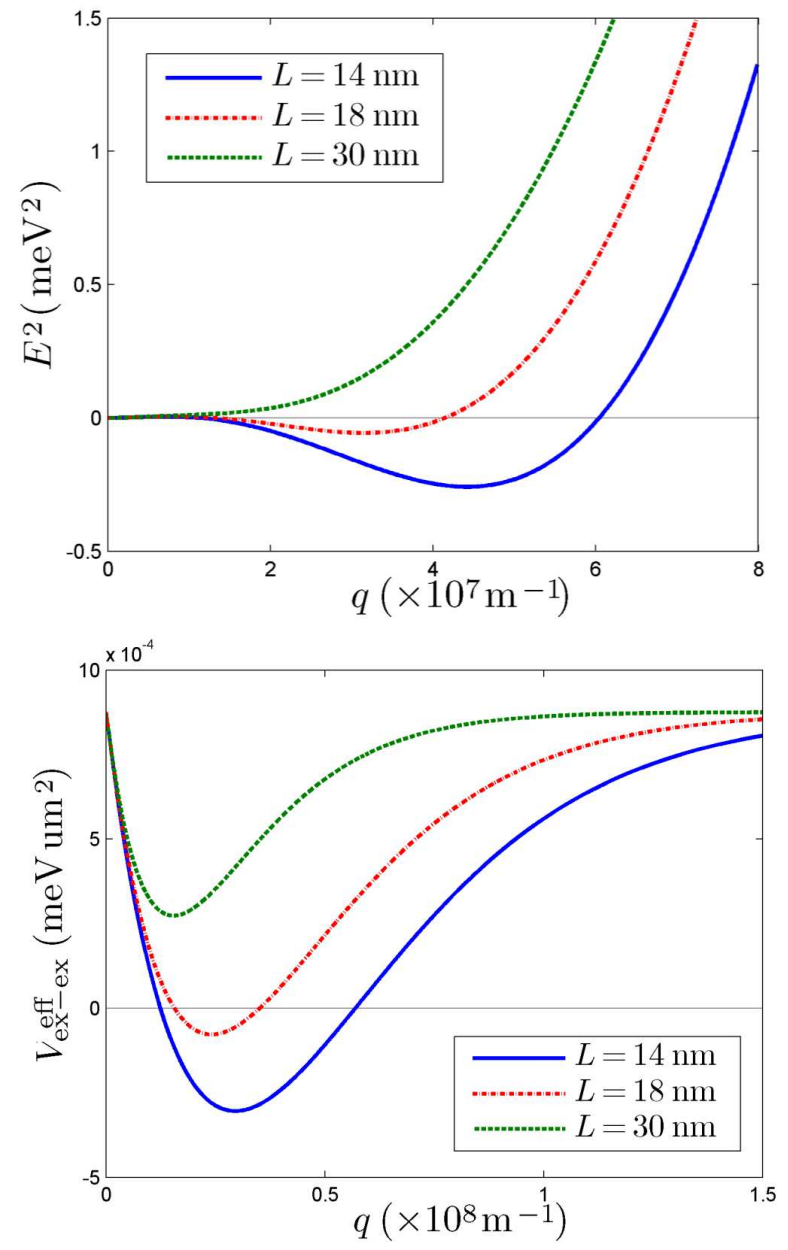

FIG. 1. The excitation spectrum (top) and effective excitonexciton interaction potential (bottom) for three values of the exciton-electron layer separation $L$. We use the exciton mass $m_{\mathrm{ex}}=0.21 m_{\mathrm{e}}$, and the average exciton density $\bar{n}_{\text {tot }}=10^{11} \mathrm{~cm}^{-2}$. The exciton dipole separation, $l=12 \mathrm{~nm}$, and the correction factor, $\xi=0.05$.

$N_{+}\left|\psi_{+}(\mathbf{r}, t)\right|^{2}+N_{-}\left|\psi_{-}(\mathbf{r}, t)\right|^{2}$, and $V_{\text {ex-ex }}^{\text {eff }}(\mathbf{r})$ is the spinisotropic exciton-exciton interaction potential. The corresponding time-dependent Gross-Pitaevskii (GP) equations for the two condensates read

$i \hbar \frac{\partial \psi_{ \pm}(\mathbf{r}, t)}{\partial t}=-\frac{\hbar^{2} \nabla^{2}}{2 m_{\mathrm{ex}}} \psi_{ \pm}(\mathbf{r}, t)+$
$+\int V_{\mathrm{ex}-\mathrm{ex}}^{\mathrm{eff}}\left(\mathbf{r}-\mathbf{r}^{\prime}\right) n_{\mathrm{tot}}\left(\mathbf{r}^{\prime}, t\right) d \mathbf{r}^{\prime} \psi_{ \pm}(\mathbf{r}, t)+\alpha n_{\mp}(\mathbf{r}, t) \psi_{ \pm}(\mathbf{r}, t)$

Derivation of the effective exciton-exciton interaction potential in this system has been recently published [10]. The matrix element of effective exciton-exciton interac- tion depending on frequency and wave-vector reads

$$
\begin{aligned}
& V_{\mathrm{ex}-\mathrm{ex}}^{\mathrm{eff}}(q, \omega) \\
& =\frac{\left(V_{22}+\frac{V_{12}^{2}(q) \Pi_{1}(q, \omega)}{1-V_{11}(q) \Pi_{1}(q, \omega)}\right)\left((\hbar \omega)^{2}-\left(E^{e x}(q)\right)^{2}\right)}{(\hbar \omega)^{2}-\left(E^{e x}(q)\right)^{2}-2 N_{0}\left[V_{22}+\frac{V_{12}^{2}(q) \Pi_{1}(q, \omega)}{1-V_{11}(q) \Pi_{1}(q, \omega)}\right] E^{e x}(q)},
\end{aligned}
$$

where

$$
\begin{gathered}
\Pi_{1}(\mathbf{q}, \omega)=\sum_{\mathbf{k}} \frac{f_{\mathbf{k}-\mathbf{q}}-f_{\mathbf{k}}}{\hbar \omega+i \hbar \delta+E_{\mathbf{k}-\mathbf{q}}^{e l}-E_{\mathbf{k}}^{e l}}, \\
V_{11}=\frac{e^{2}}{2 \epsilon_{0} \epsilon A} \cdot \frac{1}{q}
\end{gathered}
$$

with $\epsilon$ denoting the dielectric constant of the media and $A$ the sample area. The matrix element of exciton-exciton interaction $V_{22}$ is dominated by dipole-dipole repulsion [18, 19] and can be expressed as

$$
V_{22}(q)=\frac{e d \xi}{\epsilon_{0} \epsilon A}
$$

where $d$ is the exciton dipole moment in normal to the QW plane direction, $\xi$ accounts for the reduction of dipole-dipole repulsion due to electron-hole pair correlations in the exciton condensate [19]. The expression for the matrix element of electron-exciton interaction reads (the details of the calculation can be found in e.g. $\operatorname{Ref} 20)$ :

$$
\begin{aligned}
& V_{12}(q)= \\
& \quad \frac{e d e^{-q L}}{2 \epsilon_{0} \epsilon A}\left\{\frac{\beta_{e}}{\left[1+\left(\frac{\beta_{e} q a_{B}}{2}\right)^{2}\right]^{3 / 2}}+\frac{\beta_{h}}{\left[1+\left(\frac{\beta_{h} q a_{B}}{2}\right)^{2}\right]^{3 / 2}}\right\}+ \\
& \frac{e^{2} e^{-q L}}{2 \epsilon_{0} \epsilon q A}\left\{\frac{1}{\left[1+\left(\frac{\beta_{e} q a_{B}}{2}\right)^{2}\right]^{3 / 2}}-\frac{1}{\left[1+\left(\frac{\beta_{h} q a_{B}}{2}\right)^{2}\right]^{3 / 2}}\right\}
\end{aligned}
$$

where $\beta_{e, h}=m_{e, h} /\left(m_{e}+m_{h}\right)$, with $m_{e, h}$ being the effective masses of electrons and holes.

The poles of the effective potential determine the dispersions of the collective modes of the exciton system given by the equation

$$
(\hbar \omega)^{2}=\left(E^{e x}(q)\right)^{2}+2 N_{0} \tilde{V}_{22}(q) E^{e x}(q),
$$

where

$$
\tilde{V}_{22}(q)=V_{22}+V_{12}^{2}(q) \Pi_{1}(q, \omega) /\left[1-V_{11}(q) \Pi_{1}(q, \omega)\right] .
$$

In general, the exciton-exciton interaction mediated by the electron gas is a time-dependent, retarded interaction. In order to obtain a non-retarded GP equation (2), 

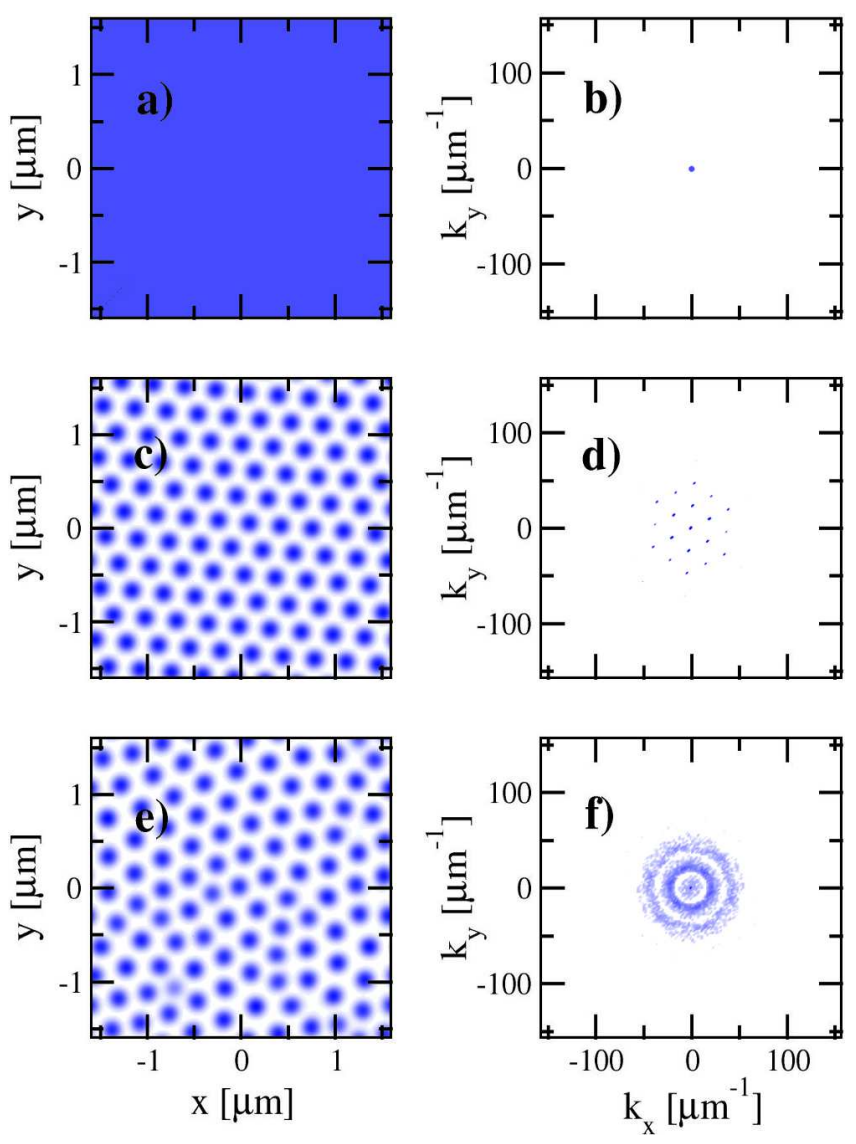

FIG. 2. Examples of density profiles of the exciton condensate in real space (left column) and reciprocal space (right column, density in logarithmic scale). The figures (a), (b) show the homogeneous ground state for separation $L=30 \mathrm{~nm}$, and the figures (c), (d) depict the supersolid ground state for $L=18 \mathrm{~nm}$, characterized by periodicity in both real and momentum spaces. The orientation of the supersolid lattice is chosen spontaneously. (e), (f) A metastable state with lattice imperfections, corresponding to a local energy minimum. In momentum space, such a state is characterized by concentric rings.

we average the potential (3) over a frequency range limited by the exciton dissociation frequency $\omega_{d}=E_{B} / \hbar$. Depending on the frequency-averaged interaction potential $V_{\mathrm{ex}-\mathrm{ex}}^{\mathrm{eff}}(\mathbf{q})$, the excitation spectrum of the condensate coupled to an electron gas can develop a roton minimum due to the attractive character of the electron mediated interactions [10], see Fig. 1. This Figure shows the exciton dispersion curves calculated from Eq. (7) in comparison with the average interaction potential for different separations $L$ between the QWs containing the 2D electron gas and the exciton condensate. Indeed, for small separation between excitons and electrons the potential becomes attractive in an intermediate range of $q$ and a roton instability develops.

The existence of a roton instability has been connected with the possible existence of a supersolid phase [1, 2], in which superfluid and crystalline orders coexist. We investigate this possibility by numerical minimization of the Hamiltonian (10) using the imaginary time propagation method. To start with we assume $\alpha=0$ and investigate a single-component exciton condensate. We determine the ground states for various physical parameters in the case of a two dimensional box with periodic boundary conditions. We take realistic values of parameters $m_{\mathrm{ex}}=0.21 m_{\mathrm{e}}, m_{\mathrm{e}}$ being the free electron mass, the average exciton density $\bar{n}_{\text {tot }}=10^{11} \mathrm{~cm}^{-2}$, with an exciton dipole separation $l=12 \mathrm{~nm}$, and different effective interaction potentials corresponding to various excitonelectron layer separation $L$.

We distinguish three regimes in which the minimization procedure leads to qualitatively different results. In the case of a positive roton gap, when the excitation spectrum does not cross into the negative energy domain, the ground state is a homogeneous superfluid, as depicted in Figs. 2(a) and (b) for $L=30 \mathrm{~nm}$. When decreasing the interlayer distance to $L=18 \mathrm{~nm}$, a dramatic change occurs, and a state characterized by a periodic triangular lattice becomes the ground state of the system, see Figs. 2(c) and (d). The orientation of the lattice is chosen randomly in a process of spontaneous symmetry breaking, depending on the initial fluctuations in the numerical minimization procedure. This symmetry breaking is additional to the symmetry breaking associated with the randomly chosen phase of the condensate wave function. In the reciprocal space, the appearance of crystalline order is manifested by the appearance of side peaks corresponding to the lattice wave vectors. We note that the lattice constant of the pattern is determined by the wave vector value at the roton minima. Indeed, the side peaks closest to $k=0$ in Fig. 2(d) match with the position of the roton minimum in Fig. 1.

When decreasing the interlayer distance even further to $L=14 \mathrm{~nm}$, the minimization algorithm leads to the collapse of the wave function $\psi_{ \pm}$into a single point of the numerical mesh. This indicates that the ground state cannot be determined due to the breakdown of the meanfield model. To obtain a correct ground state, one has to include higher order effects that would limit the growth of the local condensate density. We note that collapse is generally encountered for a nonlinear Schrödinger equation in the 2D (critical) case with attractive nonlinearity [21].

In the supersolid regime $(L=18 \mathrm{~nm})$, depending on the initial noise, the minimization procedure can produce yet another type of solution, which resembles a crystal structure with dislocations, see Fig. 2(e). While these solutions do not correspond to the ground state of the system, they are local minima of the energy and constitute metastable states, and as such are likely to appear in experimental realizations. In the momentum space, these disordered supersolid patterns are characterized by 

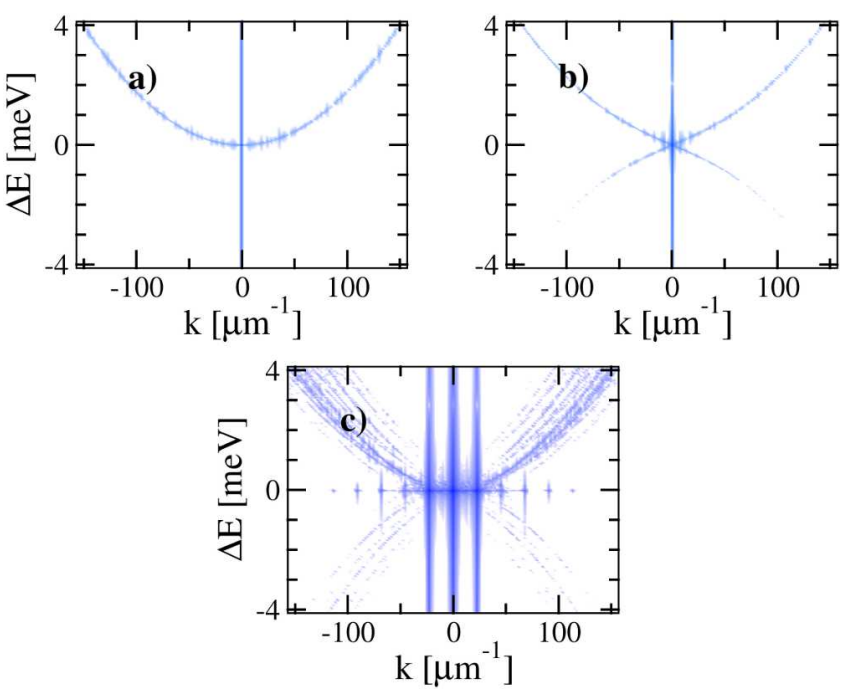

FIG. 3. Numerically determined spectra of a weakly perturbed (a) noninteracting exciton condensate, (b) condensate interacting through a local (momentum-independent) potential, and (c) supersolid condensate in the presence of coupling with electron gas, interacting through an effective potential $V_{\text {ex-ex }}^{\text {eff }}$ (parameters as in Figs. 2(c),(d)). While in the cases (a) and (b) we obtain the parabolic and linear Bogoliubov spectra as predicted by the theory, in the case (c) the spectrum is distorted as a result of interference of branches starting from different $k$-components of the ground state in the momentum space, corresponding to the vertical lines. The density is shown in a logarithmic intensity scale.

concentric rings around the $k=0$ point, see Fig. 2(f).

We also investigate the influence of the supersolid transition on a weakly excited system. In Fig. 3 we show the spectra of perturbed ground states allowed to evolve in time according to Eq. (2). We obtain the spectra by Fourier transforming the wave function $\psi_{\text {ex }}$ in space and time. The three figures correspond to the cases of a noninteracting exciton condensate, a condensate with a $q$ independent interaction potential, equivalent to a contact (local) repulsive interaction in the absence of an electron gas, and an exciton supersolid interacting through a $q$ dependent interaction potential $V_{\mathrm{ex}-\mathrm{ex}}^{\mathrm{eff}}$. While the first two figures show the corresponding free-particle parabolic spectrum and the Bogoliubov spectrum of excitations, respectively, in the case of the supersolid the spectrum becomes blurred as a result of interference of several branches starting from different points in the momentum space. These points correspond to the $k$-space components of the ground state, visible as pronounced vertical lines. The excitation branches appear to be linear close to their points of origin, which is an indication of a nonvanishing superfluid fraction.

Interesting new physics can be expected in twocomponent exciton condensates, where $\alpha \neq 0$. Fig. 4 shows an example of the spatial distribution of excitons in the supersolid phase in this case. One can see that

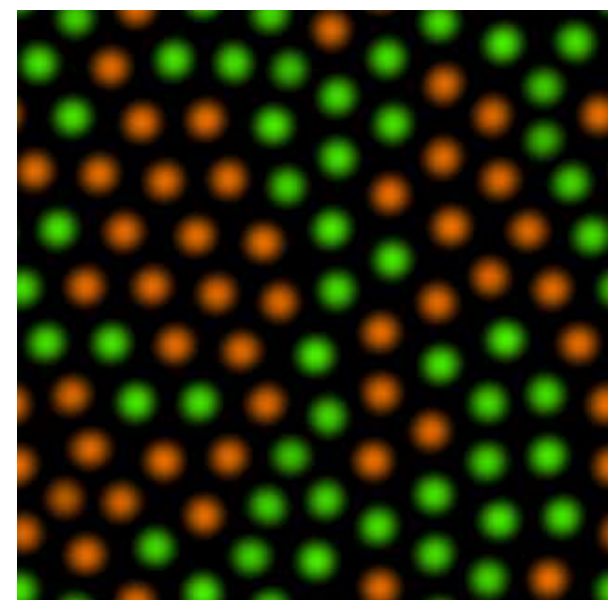

FIG. 4. Numerically calculated density plot of the exciton condensate in the supersolid phase, showing the ferromagnetic domain structure. Here $\bar{n}_{+}=\bar{n}_{-}=10^{11} \mathrm{~cm}^{-2}, L=18 \mathrm{~nm}$ and $\alpha$ is chosen in such a way that for $q=0$ the interaction of excitons with opposite spin is $10 \%$ stronger than the interaction of excitons with parallel spins. The green and orange coloring corresponds to the +1 and -1 spin components.

ferromagetic domains are formed: the triangular crystal lattice is maintained in each domain, while long-range order is broken by stochastic domain boundaries.

Experimentally, formation of the exciton supersolid may be detected by angular and polarisation resolved photoluminescence, which would give access to the characteristic diffraction pattern (most likely, concentric rings) in the Fourier spectrum of excitations of the supersolid.

M.M. acknowledges support from the Foundation for Polish Science through the "Homing Plus" programme. TT and AK thank Ivan Shelykh for useful discussions.

[1] A. F. Andreev and I. M. Lifshitz, Sov. Phys. JETP 29 (1969) 1107; G.V. Chester, Phys. Rev. A 2, 256 (1970); A. J. Leggett, Phys. Rev. Lett. 25, 1543 (1970).

[2] N. Henkel, R. Nath, and T. Pohl, Phys. Rev. Lett. 104, 195302 (2010); F. Cinti et al., Phys. Rev. Lett. 105, 135301 (2010); M. Matuszewski, Phys. Rev. Lett. 105, 020405 (2010).

[3] E. Kim and M.H.W. Chan, Nature (London) 427, 225 (2004); E. Kim and M.H.W. Chan, Science 305, 1941 (2004); D. E. Galli and L. Reatto, J. Phys. Soc. Jpn. 77, 111010 (2008); S. Balibar, Nature (London) 464, 176 (2010).

[4] A. E. Golomedov, G. E. Astrakharchik, and Yu. E. Lozovik, Phys. Rev. A 84, 033615 (2011).

[5] L.V. Butov, C.W. Lai, A.L. Ivanov, A.C. Gossard, and D.S. Chemla, Nature (London) 417, 47 (2002).

[6] J. Kasprzak et al., Nature (London), 443, 409 (2006); R. Balili, V. Hartwell, D. Snoke, L. Pfeiffer and K. West, Science, 316, 1007 (2007). 
[7] A V Kavokin, J J Baumberg, G Malpuech, F P Laussy, Microcavities, Oxford University Press (2007).

[8] A. Amo et al., Nature (London) 457, 291 (2009).

[9] C. Ciuti et al., Phys. Rev. B 58, 7926 (1998).

[10] I. A. Shelykh, T. Taylor, and A. V. Kavokin, Phys. Rev. Lett. 105, 140402 (2010).

[11] M.M. Parish, F.M. Marchetti and P.B. Littlewood, Europhysics Letters, 95, 27007 (2011).

[12] Yu. E. Lozovik and V. I. Yudson, Pis'ma Zh. Eksp. Teor.Fiz. 22, 556 (1975) [JETP Lett. 22, 274 (1975)]; R. Zimmermann, Phys. Status Solidi B 243, 2358 (2006); Z. Vörös, D. W. Snoke, L. Pfeiffer, and K. West, Phys. Rev. Lett. 103, 016403 (2009).
[13] V.I. Yudson, Phys. Rev. Lett. 77, 1564 (1996).

[14] F.P. Laussy, A.V. Kavokin and I.A. Shelykh, Phys. Rev. Lett. 104, 106402 (2010).

[15] M. Combescot, O. Betbeder-Matibet, and R. Combescot, Phys. Rev. Lett. 99, 176403 (2007).

[16] N. W. Sinclair et al., Phys. Rev. B 83, 245304 (2011).

[17] A. J. Leggett, Rev. Mod. Phys. 73, 307 (2001).

[18] B. Laikhtman and R. Rapaport, Phys. Rev. B 80, 195313 (2009).

[19] C. Schindler and R. Zimmermann, Phys. Rev. B 78, 045313 (2008).

[20] G. Ramon et al., Phys. Rev. B 65, 085323 (2002).

[21] L. Bergé, Physics Reports 303, 259 (1998). 